\section{IMPLANTACIÓN DE LA EDUCACIÓN BASICA, AUGE Y CRISIS DEL PROYECTO 1979-1989}

\section{Blanca Rojas de Chirinos Carmen Yaquelina Reyes Leal} UPEL-IPB

Recibido :23-03-06

Aceptado:02-11-06

\section{RESUMEN}

Este artículo resume los resultados de una investigación que tuvo como propósito comprender bajo un criterio de aproximación sobre la base de tres variables: Estado, Ideología y Educación, los cambios en el sistema educativo venezolano como producto de la reforma educativa fundamentada en el concepto de Estado Promotor, que se sustentaba según Urbaneja D (1985) en: un principio socialcristiano llamado el principio de subsidiariedad, según el cual el Estado debe dejar que las sociedades intermedias lleven a cabo lo que puedan realizar por sí mismas. En atención a la estrategia de recolección de información, el estudio se inscribe en la modalidad de investigación documental que en atención a la disciplina corresponde a una investigación histórica de carácter interpretativo que se ubica en la perspectiva sociohistórica de la educación. El proceso de investigación se inició con un arqueo de fuentes con la finalidad de precisar información referente al tiempo y espacio en el cual se dio la Reforma Educativa. En la medida que se recopiló la información, se levantó una matriz de análisis donde se registraron los datos sobre el contexto sociopolítico; los fundamentos ideológicos-políticos de la Escuela, los cambios curriculares más significativos y la evolución histórico-legal de la supervisión educativa en Venezuela correspondiente a la tercera década de la Democracia Venezolana comprendida entre 1979 y 1989. Los resultados de la investigación evidencian que esta etapa culmina con el planteamiento de una propuesta de descentralización limitada y progresiva, dirigida en un primer momento sólo al nivel preescolar y básico, con énfasis en una transferencia futura en primero a los municipios y de segundo a los estados.

Palabras claves: Estado, ideología, educación básica, reforma educativa

\section{BASIC EDUCATION FOUNDATION, PEAK AND CRISIS OF} THE 1979-1989 PROJECT

\section{ABSTRACT}

This research was to understand, under an approach criteria based on three variables state, ideology and education, the venezuelan educational system changes as a product of an educational reform supported in the concept of Urbaneja (1985) Promoter State. The study is a documental and historical of interpretative type one. The research process was initiated with a search of sources to determine information referred to time and space in which the Educational Reform was given. Next, an analysis matrix was done in which data about socio-political context were registered; the ideological-political basis of school, the most meaningful curricular changes and the historical-legal evolution of educational supervising in Venezuela related to the third decade of the Venezuelan Democracy (1979 and 1989). The outcomes of the research demonstrate that this stage ends with the approach of a proposal of a limited and progressive non centralism, aimed at the preschool and basic stage only at the first moment, emphasizing in a future transference to the municipalities firstly and to the states secondly.

Key words: State, ideology, educational reform.

\section{INTRODUCCIÓN}

Al explorar el contexto ideológico en el cual se han dado las reformas en la Educación Venezolana y en especial en la Escuela Primaria y Básica entre 1948 y 1998, se aprecia que los constantes cambios legislativos que han impulsado la reforma educativa han estado muy relacionados con el sistema sociopolítico en cada período histórico.

En contribución al estudio de esta situación problema, surge esta ponencia que es producto de una investigación documental, tipo histórica, de carácter interpretativo que se realizó con el propósito de comprender los cambios que se generaron en la Escuela Primaria, bajo un criterio de aproximación a la relación de tres variables: Estado, ideología y educación, desde 1979 hasta 1989. En atención a la estrategia metodológica empleada en esta modalidad indagatoria, el proceso de investigación se inició con un arqueo de fuentes con la finalidad de precisar información referente al tiempo y espacio en el cual se dio la Reforma Educativa fundamentada en el concepto de “Estado Promotor” que se sustentaba según Urbaneja D (1985) en: “un principio socialcristiano llamado el principio de subsidiariedad, según 
el cual el Estado debe dejar que las 'sociedades intermedias' lleven a cabo lo que puedan realizar por sí mismas” p.236.

En la medida que se recopiló la información, se levantó una matriz de análisis donde se registraron los datos sobre el contexto sociopolítico; los fundamentos ideológicos-políticos de la Escuela, los cambios curriculares más significativos y la evolución histórico-legal de la supervisión educativa en Venezuela correspondiente a la tercera década de la Democracia Venezolana. Estos aspectos se describen a continuación en el cuerpo de la ponencia.

\section{CONTEXTO SOCIOPOLÍTICO DE LA REFORMA \\ EDUCATIVA 1979-1989: TERCERA DÉCADA DE LA DEMOCRACIA: DETERIORO Y DESAFÍOS.}

En esta década continuó fortaleciéndose el bipartidismo, bajo la administración del Partido Socialcristiano COPEI, con la presidencia de Luis Herrera Campins (1979-1984), seguido de Jaime Lusinchi (1984-1989) de Acción Democrática. Por el Ministerio de Educación pasaron seis ministros: Rafael Hernández Heres (1979-1982), Felipe Montilla (1982-1984), Ruth Lerner de Almea (1984-1985), Luis Manuel Carbonell (1985-1986).Pedro Cabello (1986-1987) y Laura Castillo de Garfinkel (1988-1989).

Tal como se observa en el segundo quinquenio los ministros de educación permanecieron por espacio de un promedio de quince meses frente al despacho, circunstancia que impidió darle continuidad a la labor emprendida en materia educativa, cuyos resultados se recogen a largo plazo y los errores son de difícil enmienda a corto tiempo.

Esta tercera década de la democracia, representa para la sociedad venezolana un momento de súbita transformación: el advenimiento de la cuarta revolución (La primera revolución representó el contacto con la cultura hispánica; la segunda, la liberación política; la tercera, el ingreso de nuestra sociedad a la economía petrolera y a la cultura norteamericana), que representa la imposibilidad de continuar subsidiando un modelo de sociedad en expansión, sin una base real de producción.

Es decir, el fin de la sociedad en donde el ingreso petrolero podía sustentar una administración dependiosa y en efecto relativamente ficticia, donde se manejaban ingresos que no se producían directamente. Razón por la cual se denomina a esta cuarta revolución, la Venezuela postpetrolera, denominación que según Albornoz (1991):

Oculta el hecho de que el drama esencial de esta cuarta revolución consiste, justamente, en que si bien el petróleo continuará siendo el eje de nuestra economía, los ingresos provenientes por dicho renglón son insuficientes para abordar con éxito, aparentemente, las cargas internas y externas que gravitan sobre nuestra economía. P. 95.

En consecuencia la década de los ochenta se constituyó en el inicio de un cambio revolucionario en la sociedad venezolana, oportunidad en la cual se produjeron desajustes y desequilibrios que impulsaron cambios radicales en educación, orientados fundamentalmente a superar el modelo educativo de la Venezuela del Subsidio, de la Venezuela de la Tercera Revolución, dado que tanto para nuestro país como para el resto de los países de América Latina, esta década, constituyó un período de decrecimiento económico, durante el cual el ingreso real por habitante retrocedió a los niveles de 1977 hasta tal punto que según Rodríguez (1998): "La Organización Internacional del Trabajo, la consideró como la región con la mayor deuda externa en el tercer mundo, el mayor éxodo rural-urbano, el mayor crecimiento de la marginalidad y la pobreza y la mayor concentración de la tierra en pocas manos.” P. 267

Desde el punto de vista político la crisis de los ochenta estuvo acompañada de una excesiva concentración del poder en manos de los dos partidos políticos mayoritarios, impidiendo la participación de la 
población en la toma de decisiones. El clientelismo partidista invadió todos los sectores de la actividad económica, las instituciones financieras, los ministerios del poder ejecutivo y el poder judicial; fomentando la incapacidad administrativa y la corrupción en el manejo de los fondos.

La crisis profundizó las diferencias, ya existentes, entre los grupos que componen nuestra sociedad. Acelerando según la autora antes mencionada, por una parte, la concentración de la riqueza en grupos financieros y rentistas; y por la otra, generando el empobrecimiento de la clase media y aumentando el porcentaje de la población en estado de pobreza, estimado para 1990 de 36\% y de pobreza crítica en 43\%, lo cual trajo como consecuencia el incremento de la deserción escolar, delincuencia juvenil, mendicidad, drogadicción y otras formas de degradación del ser humano, de dónde se derivó una crisis ética, cuyo centro es la desvalorización de la dignidad humana, en contrapartida a la exaltación del poder y el dinero, sin importar los medios para su obtención.

Dentro de ese contexto político, económico y social antes descrito, se desarrolla esta tercera década de la democracia cuyos lineamientos en materia educativa quedan explicitados en el VI y VII Planes de la Nación que fueron elaborados en correspondencia cronológica con ambos períodos presidenciales, los cuales serán descritos en los quinquenios correspondientes.

\section{Quinquenio 1979-1984}

En este quinquenio la sociedad venezolana, presenta un conjunto de problemas que demandaban la rectificación del modelo de desarrollo económico escogido, haciéndolo más redistributivo, mejorando las condiciones y calidad de vida del venezolano e incrementando al mismo tiempo, la eficiencia del sistema económico.
Desde esa nueva concepción del desarrollo, se propuso el Plan de Desarrollo global de la Nación, mediante el cual la educación fue establecida como una prioridad social dada su capacidad para activar los procesos de transformación de los pueblos y las realizaciones individuales y colectivas, para propiciar la igualdad social y afirmar la identidad nacional, alentando el desarrollo político de la nación y su proceso de maduración histórica.

Desde otro punto de vista, el crecimiento y la transformación de la economía demandaba un mayor esfuerzo en la formación de recursos humanos calificados a través de la educación y la capacitación técnica, así como un mayor estímulo a la creación científica, tecnológica y a los procesos de difusión del conocimiento.

En sí, la situación del sector educativo indicaba que esta década (1979-1984), se iniciaba con problemas en la calidad y pertinencia de la educación y de la formación para el trabajo, problemas en la cobertura de los servicios educativos y problemas en la gestión administrativa del sector.

La magnitud y complejidad de los problemas antes señalados, exigían que su tratamiento y búsqueda de soluciones, debía abordarse mediante un enfoque estratégico que permitiera por una parte adecuar la estructura y funcionamiento del sistema educativo venezolano a las expectativas de la sociedad y por otra, orientar la política educacional en atención a cuatro principios básicos: la democratización, para responder a la necesidad de un acceso equitativo a las oportunidades de educación; la diversificación, para armonizar las oportunidades ofrecidas con las necesidades y demandas individuales y de los grupos sociales; la regionalización, para que estas oportunidades estuviesen en correspondencia con los intereses de las variables características regionales; y la participación, dado que uno de los objetivos fundamentales del VI Plan de la Nación era el fortalecimiento del sistema democrático a través de la promoción de una sociedad participativa, con la presencia activa del pueblo tanto de las personas 
como de las organizaciones, en la preparación, toma y ejecución de decisiones, de tal forma que desarrollaran responsablemente sus iniciativas, entregaran sus aportes y recibieran una justa parte de los beneficios del esfuerzo social.

Sobre la base de estos principios, las acciones de mejoramiento cualitativo y enriquecimiento de la educación tendrían como propósito fundamental: incentivar el conjunto de la población venezolana a desarrollar todas sus potencialidades y, como consecuencia de ello, estimular la creación y promoción de condiciones que hicieran más factible la vigencia de un orden social más justo, regido por el derecho y animado por la participación consciente, solidaria y para lograr el desarrollo nacional y regional en un clima de paz, libertad y convivencia.

COPEI, durante este quinquenio fundamentó su política educativa en el concepto de "Estado Promotor" que se sustentaba según Urbaneja D (1985) en: "un principio socialcristiano llamado el principio de subsidiariedad, según el cual el Estado debe dejar que las 'sociedades intermedias’ lleven a cabo lo que puedan realizar por sí mismas” p.236.

Dentro de esa concepción ideológica se buscaba el desarrollo de una sociedad y un ciudadano más autogestionario y menos dependiente del Estado, se le daba continuidad a las políticas de estado iniciadas en 1969 bajo la presidencia de Rafael Caldera.

Todas estas ideas fueron incorporadas al diseño curricular del subsistema de la Educación Básica de nueve grados que se creó el 13 de Junio de 1980, mediante el Decreto No 646 y entró en vigencia el 26 de Julio de ese mismo año con la promulgación de la Ley Orgánica de Educación, al quedar legalmente institucionalizada según los artículos 16 y 21 de la mencionada ley.

Los principios fundamentales en los cuales se sustentaba el referido diseño curricular eran: democratización, participación, autorrealización, creatividad, identidad nacional, regionalización y desarrollo autónomo. Lo que apuntaba según Rodríguez N (1998) “a la promoción de la participación de la población en la toma de decisiones y ejecución de acciones para mejorar la vida en las comunidades”. P. 271.

En sí, la política de regionalización y participación, proponía la descentralización en la toma de decisiones y la desconcentración en la prestación de algunos servicios. Se promovía a la vez un cierto grado de autonomía en las escuelas, para establecer una normativa interna y tomar algunas decisiones en relación al currículo, de allí la creación de los comités de planes y programas en cada plantel. En este sentido con la promulgación de la Ley Orgánica de Educación de 1980 (Gaceta Oficial No 2.635 de fecha 16 de Julio de 1980), se intentaba hacer más pertinente el Sistema Educativo Venezolano con las necesidades individuales y sociales del país, mediante la incorporación como aspecto innovador, la Educación para el Trabajo en todos los niveles de la Educación Básica.

Se concibe una educación donde la escuela opere al mismo ritmo de la comunidad, de manera permanente y adecuada a la ecología propia de la región, admitiendo que la práctica escolar es una microsolución que debe partir de la experiencia cotidiana de los actores e instrumentos del proceso de enseñanza y aprendizaje. Tal como lo plantea Albornoz (1991), la comunidad debe tener el control de la escolaridad con capacidad de decisión para influir en el diseño curricular, en la selección de los maestros, de los textos, de los equipos y actividades y sobre todo capacidad para emplear la unidad escolar como gestor del cambio y la transformación social.

Esa concepción de la función de la escuela en el proceso de cambio y transformación de las comunidades, exigía del docente el desempeño en diferentes roles que debían ser definidos. En atención a ello el Ministerio de Educación emite la resolución No 12 que describe el perfil del docente en atención a cinco roles básicos: facilitador del 
aprendizaje, investigador, orientador, promotor social y administrador. Con el rol de promotor social se aspiraba la intervención del docente en la "integración escuela-comunidad, al propiciar la participación y la conjugación de esfuerzos que contribuyan a satisfacer necesidades socio-culturales y educativas de la comunidad..."

Frente a todas esas exigencias que demandaba la implantación del modelo normativo del Proyecto Experimental de Educación Básica, el Ministerio de Educación emprendió una serie de acciones dirigidas a producir cambios sustanciales en la estructura de áreas, programas de estudio, estrategias de aprendizaje y sistemas de evaluación. Incorporó componentes curriculares que respondieran a las características y necesidades específicas de la comunidad regional. Consideró a sí mismo un proceso de actualización y sensibilización de los docentes y una mayor coordinación interinstitucional, participación y utilización de los recursos de la comunidad.

Estas innovaciones estuvieron influenciadas directamente por la Declaración de México, documento de la Conferencia Regional de Ministros de Educación y Ministros encargados de la Planificación económica de los Estados Miembros de América Latina y del Caribe, convocada por la UNESCO y reunida en México del 4 al 13 de diciembre de 1979 .

En dicha conferencia se llegó a varios acuerdos entre los cuales cabe señalar el referido a que los Estados Miembros, entre ellos Venezuela debía incorporar al sistema a todos los niños en edad de escolaridad obligatoria antes de 1999, e iniciar a los alumnos especialmente los pertenecientes a los sectores pobres desde temprana edad en la valoración y práctica del trabajo.

En concordancia con los planteamientos del mencionado documento, el nivel de Educación Básica fue sometido a dos reformas del currículo en esta tercera década de la democracia (1979-1989), cada una liderizada por el gobierno de turno, tal vez sea esa una de las razones por la cual ninguna de las reformas logró cambios visibles en el mejoramiento cualitativo de la enseñanza, en los resultados del aprendizaje y ni en el aumento de la prosecución escolar hasta el último grado de la segunda etapa de Educación Básica y menos aún en el último de la tercera etapa de ese nivel. En el primer proyecto correspondiente a este primer quinquenio de la Tercera Década de la Democracia, se dividía el referido nivel en tres ciclos, diferenciados entre sí por grado de interrelación entre las asignaturas, los énfasis curriculares y los componentes afectivos.

Desde esa concepción del currículo, la implantación de la Educación Básica se inició mediante un ensayo denominado "Proyecto Experimental de Educación Básica (1980-1983)” que se concibió en forma bastante ambicioso y poco realista. Se involucró a un número muy grande de escuelas (1445 distribuidas por todo el país) y ello hizo muy difícil manejar, controlar y hacer un seguimiento operativo del ensayo. Por otra parte los docentes que participaron en el proyecto, casi en su totalidad fueron formados en las escuelas normales de conformidad con los requerimientos y con la concepción de la Educación Primaria previstos en la Ley Orgánica de Educación de 1955, razón por la cual en su mayoría desconocían las bases conceptuales y teóricas del sistema de objetivos dominados y no dominados que se adoptó para la evaluación del rendimiento estudiantil.

La división del nivel en tres ciclos, requería la existencia de aulas disponibles en cada escuela para incorporar el séptimo grado, así como de equipos de laboratorio y otros materiales que se encontraban instalados en los institutos de Educación Media. Por esta razón durante este quinquenio (1979-1984) se empezaron a decretar y a construir edificios apropiados que sirvieran de sede a las nuevas escuelas de Educación Básica de nueve grados, adaptados y dotados conforme a la nueva concepción y orientación, lo cual trajo como consecuencia que las edificaciones de las antiguas escuelas primarias y las edificaciones 
debidamente dotadas para el funcionamiento del ciclo Básico Común quedarían sin uso.

En sí, de haberse adoptado como política la sustitución por las nuevas edificaciones, hubiese significado la inversión de cantidades considerables de dinero por este concepto, lo cual probablemente hubiese constituido un despilfarro enorme de recursos que de por sí, ya el país era imposible sostener, dado que a partir de 1982 la situación económica empezó a deteriorarse cuando se inició una crisis económica en el país que condujo al llamado viernes negro (18-02-82) y que aún hoy en día no se ha superado totalmente.

Es decir que para el momento en que se adquirieron los compromisos establecidos en la Ley Orgánica de Educación en su artículo 21, referido a la creación del nivel de Educación Básica obligatoria de nueve grados como mínimo, el Estado contaba con amplios recursos financieros, debido al aumento considerable que experimentaron los precios del petróleo como principal producto de exportación del país que generaba el $70 \%$ de los recursos del presupuesto de la nación, alcanzando para finales del año 81, más de \$35 por barril.

No obstante para el momento de implantar y poner en funcionamiento esas modificaciones antes descritas, dichos recursos se habían mermado en forma considerable, sin embargo la implantación del nivel de Educación Básica se hizo realidad en todo el país. El Estado en lo fundamental respondió con los compromisos contraídos, aún en los momentos de crisis siempre hubo una excepción: La Educación Básica.

El Ministerio de Educación solicitó y obtuvo la colaboración de los organismos internacionales y nacionales para realizar algunas acciones, tales como talleres, seminarios y reuniones de trabajo que fueron financiadas por la UNESCO, por la OEA y, por otros organismos. El apoyo de todas las instituciones de educación superior que forman docentes y en especial la Universidad Pedagógica Experimental Libertador fue muy importante en lo que se refiere a la formación y capacitación de los recursos humanos que se requerían.

Se impuso una filosofía realista que evitó traumas y frustraciones, la cual quedó muy bien definida por una expresión utilizada frecuentemente por la Viceministra de Educación, Profesora Laura Castillo Garfinkel: "Implantaremos la Educación Básica con los planteles con que cuenta el país, con los maestros que tenemos y con los recursos financieros de que disponemos” El Nacional (2-12-81) D. p.7.

Finalmente la división de la Escuela Básica que se adoptó y generalizó para todo el país entre 1985 y 1987 comprendió tres etapas, de las cuales las dos primeras siguieron funcionando en los planteles que ocupaban las escuelas primarias, mientras que la tercera etapa utilizó las edificaciones que ocupaban anteriormente los ciclos básicos. Esta decisión se fundamentó en factores económicos y en la evaluación de la experiencia del ensayo que se había realizado en las 1445 escuelas del país a las cuales se hizo referencia anteriormente.

Por otra parte durante este quinquenio, otro aspecto que no se extendió de manera obligatoria fue el sistema de planificación que se adoptó a manera de ensayo, denominado "Unidad Generadora de Aprendizaje” (UGA), basado en los centros de interés de Ovidio Decroly, que en cierta forma se venía ensayando en Venezuela desde la década de los treinta dentro de la concepción de la Escuela Nueva.

En la unidad Generadora de Aprendizaje se sintetizaba la planificación completa del proceso. En ella ase planteaban los objetivos tanto locales como nacionales que eran asumidos a partir de un eje de interés propuesto por los alumnos. Igualmente a través de la UGA se planificaban las estrategias metodológicas, los contenidos, las actividades, los recursos y la evaluación. Toda esta planificación tiene un carácter globalizador, debido a que se intentaba interrelacionar las 
diversas áreas para que formaran parte de un mismo proceso, respondiendo a un mismo eje de interés.

Es importante destacar otros aspectos a los cuales se les dio especial tratamiento durante este quinquenio, tal es el caso de la creación del sistema de servicios bibliotecarios escolares según Decreto No 188 de fecha 27-06-79. Se creó también el seguro escolar por Decreto No 357 de fecha 15 de Noviembre de ese mismo año para los menores inscritos en planteles oficiales de preescolar y Educación Básica y en las mismas instituciones privadas que atendían a menores de las zonas de bajos recursos.

\section{Quinquenio 1984-1989}

Durante la gestión del Presidente Jaime Lusinchi se designó la Comisión Presidencial para el Estudio del Proyecto Educativo Nacional (COPEN), coordinada por Arturo Uslar Pietri e integrada por un grupo de ciudadanos, todos de altos credenciales personales y profesionales, expertos en materia educativa, de distintas tendencias ideológicas y pareceres, entre los cuales caben mencionar los siguientes: Orlando Albornoz, Felipe Bezara, Antonio Luis Cárdenas, Germán carrera Damas, Senta Essenfeld, Edmundo Chirinos, Arnoldo Gabaldón, Ignacio Iribarren, Iván Olaizola, Enrique Pérez Olivares, Luis Beltrán Prieto Figueroa y Pedro Rincón Gutiérrez, todos bajo la coordinación de ese ilustre venezolano Arturo Uslar Pietri.

Esta comisión muy responsablemente, asumiendo su compromiso ante el país, después de recabar toda la información disponible sobre la materia en franca cooperación con el Ministerio de Educación y sus dependencias, así como de numerosas instituciones, gremios y personalidades vinculadas a la educación, analizaron con una actitud serena y objetiva la situación presente en nuestro sistema educativo, sus fallas y aciertos. Formularon además una serie de recomendaciones inaplazables, necesarias para rectificar, replantear y encaminar la educación venezolana al logro de sus verdaderos fines.
En sí esta comisión designada por disposición del Presidente de la República, según Resolución No 154 emitida por el Ministerio de Educación el 14 de Marzo de 1985, realizó un estudio prospectivo de todo el sistema educativo venezolano durante diecisiete meses y más de setenta reuniones periódicas de trabajo, el cual lo plasmaron en el Informe de la Comisión Presidencial para el estudio del Proyecto Educativo Venezolano, publicado en 1986.

Este informe consta de dos partes: una primera que contiene una visión general de la educación venezolana, referida a la apreciación general de sus problemas más importantes para ese entonces. La segunda contiene las recomendaciones generales que a juicio de la comisión servirían de fundamento para corregir los defectos de la educación en todos sus niveles y modalidades y orientarla de modo que sirva a los fines supremos de la República, partiendo de los principios rectores de la educación venezolana tal como estaban establecidos en la Constitución Nacional de la República de Venezuela promulgada en 1961.

Todo esto trajo como consecuencia la reactivación del Consejo Nacional de Educación, organismo asesor del Ministerio de Educación creado en 1974 y había permanecido inactivo, decretándose su nueva organización en Julio de 1986. Su actividad en primera instancia estuvo dirigida a responder al informe de la Comisión Presidencial para el Estudio del Proyecto Educativo Nacional (COPEN, 1986) y en segunda instancia al análisis de los proyectos educativos que fueron presentados en el país en la década de los ochenta, entre los cuales caben señalar: Educación en Venezuela: problemas y soluciones (COPEN,1986); "Hacia un Proyecto Educativo Nacional (UCV,1986); "Proyecto Educativo de la Asociación Venezolana de la Educación Católica (AVEC,1986); y "Un Proyecto Educativo para la Modernización y la Democratización (COPRE,1990), culminando en 1993 con el Plan Decenal del Consejo Nacional de Educación y un informe del Banco Mundial. 
Ahora bien en cuanto a la política en materia de educación durante este quinquenio, está contenida en el VII Plan de la Nación (19841988), diseñado según los procedimientos de la planificación estratégica, restándole importancia a las directrices orientadoras generales, por ejemplo el Proyecto estratégico No 10"Acceso a la educación y a la cultura”, que partía del siguiente objetivo:

Consolidar las bases para la formación de un ciudadano, creativo, crítico e integral en el marco de una democracia más participativa. Este será un proceso continuo y persistente que debe ser perseguido y realizado con tenacidad. Para ello es necesario una educación que responda a las necesidades colectivas mediante una organización más moderna y eficiente, que permita una mayor igualdad de oportunidades tanto en el acceso y permanencia en el sistema educativo, como en la incorporación al mercado de trabajo. (pág. 6)

En sí, estaba en correspondencia con el gran proyecto nacional: la modernización, cuyo fin último era la creación de una sociedad moderna e industrializada, el cual le adjudicaba a la educación un papel fundamental dentro de ese proceso modernizador: "darle a la población herramientas para insertarse como mano de obra calificada en la nueva economía moderna” González (1996, pág. 15).

Al respecto, es importante acotar que el Estado Venezolano, le adjudicaba esa función a la educación, dentro de esa política social tradicional que había caracterizado al modelo de Estado de Bienestar que desde 1958 había imperado en el país, mediante una educación gratuita en todos sus niveles y modalidades, servicios de salud prestados gratuitamente, subsidios a los principales servicios básicos (agua, electricidad, gasolina, gas, teléfono, entre otros) y a una gran variedad de productos de consumo masivo (alimentos sobre todo); control de mercado laboral y de los precios y, establecimiento de un sistema de seguros sociales para proteger a la población trabajadora.

Es decir, una política que no facilitaba la transformación de la sociedad venezolana en un nuevo escenario de país moderno y productivo, sino por el contrario sólo se lograba paliar los efectos de la ineficiencia y la improductividad de las empresas nacionales, debido a que no se le daba a las políticas sociales suficiente importancia como posibles transformadoras de la sociedad venezolana, pues se suponía que el crecimiento económico con su dinámica propia por sí mismo, lograría el cambio al generar suficientes empleos como para insertar a toda la población y erradicar la pobreza del panorama nacional.

La política social, concebida desde ese punto de vista, no intentaba generar condiciones que favorecieran el trabajo y la productividad, dado que la relación entre el Estado y el beneficiario se reducía a un vínculo clientelista, paternalista y de dependencia. En definitiva, esa política, sólo estaba destinada a transferir incondicionalmente recursos a la población mientras ocurría la modernización desde la vía económica.

A pesar de los aspectos negativos que se mencionaron anteriormente de estas políticas, la ejecución de las mismas dio pronto sus resultados: el incremento constante de la matrícula no sólo en Educación Básica sino en todos los niveles del sistema educativo venezolano, es decir, la masificación fue un éxito desde el punto de vista de la cobertura.

El resultado global de las políticas de salud instrumentadas fue un constante aumento de la esperanza de vida de los venezolanos, a pesar existían fallas en el sistema para cubrir a la población en las distintas regiones del país. La masificación de la seguridad social fue otro de los indicadores, producto de la política social tradicional, cuando a partir de 1966 se extiende, el alcance de los seguros sociales con la nueva Ley del Seguro Social, al menos en términos normativos hacia 
toda la población trabajadora, incluyendo los riesgos a largo plazo tales como pensiones de invalidez, y vejez entre otros.

A pesar de las mejorías que mostraron los indicadores antes señalados en las primeras dos décadas de la democracia, no se logró la erradicación de la pobreza, debido a las debilidades intrínsecas de este modelo de intervención social , ya que fue imposible incluir a toda la población dentro de los bienes y servicios que ofrecía la acción del Estado. Debilidades se hicieron más evidentes con la posterior crisis de los años ochenta, cuando la escasez de recursos impidió que la política social pudiera seguir creciendo los ritmos experimentados en las décadas anteriores.

En consecuencia, estas debilidades potenciadas por la crisis económica de los años ochenta, generaron las grandes deficiencias que comenzaron a observarse en los servicios públicos, lo cual ocasionó una disminución de la calidad de vida que habían logrado en promedio los venezolanos hasta los años setenta.

En sí, la política social tradicional no logró alcanzar los objetivos de dotar a toda la población de los servicios básicos para garantizar su acceso al desarrollo económico y el bienestar mediante un empleo productivo. En primer lugar porque grandes sectores de la población se encontraban excluidos de las redes sociales del Estado. En segundo lugar porque el servicio prestado era deficiente: el sistema educativo no estaba preparando ciudadanos para el trabajo productivo y el sistema de salud no podía responder con calidad a las necesidades de la población.

A estos problemas generados por la escasez de recursos, se sumó el de la política social tradicional que había sido incapaz de enfrentar la crisis por su inflexibilidad y su incapacidad para adaptarse a las circunstancias cambiantes vividas en el país en los últimos años.
La concentración de los recursos y la inelasticidad del gasto en las áreas tradicionales de atención (educación y salud fundamentalmente), estaban muy vinculadas al problema del gremialismo y del clientelismo existente en las instituciones del sector social, lo que trajo como consecuencia la aparición dentro de las agencias gubernamentales de derechos adquiridos que dificultaban cualquier intento de cambio en la asignación de los recursos.

Aunado a todo ello, el crecimiento económico y la industrialización sobre las cuales se basaban las 'esperanzas de transformación y modernización del país’, durante este quinquenio, tampoco aportaron los resultados sociales deseados, generando una serie de problemas que trajeron consigo un conjunto de medidas de austeridad pública acompañada entre otras por el aumento del precio de la carne y de la gasolina; reorganización de ciertas empresas y la liquidación de otras, tal fue el caso de la Corporación de Mercadeo Agrícola y la Corporación Venezolana de fomento (CVF). Eliminación del subsidio a la producción láctea, lo cual trajo como consecuencia el aumento del precio de estos productos.

Aparte de los hechos que la prensa señalaba diariamente: los pozos de la muerte, la matraca parlamentaria, los jóvenes asesinados en Yumare, los desaciertos en lo económico contribuyeron a que el mismo gobierno devaluara el bolívar a una paridad de más de 14 bolívares por dólar. Todo ello, aunado al incumplimiento del Pacto de Punto Fijo, al empeño del presidente en arreglar el pago de la deuda externa que confrontaba Venezuela, tanto pública como privada, y a su complacencia con los poderosos económicamente, mereció un juicio popular “el gobierno favorece a los ricos” (Alviárez;1987, pág 257 )

\section{FUNDAMENTACIÓN HISTÓRICA Y LEGAL DE LA EDUCACIÓN BÁSICA 1979-1989}

En el año de 1979 se celebra en México la Conferencia de Ministros de Educación del área de América Latina y el Caribe, en la 
cual los países asistentes y entre ellos Venezuela se comprometen entre cosas a "ofrecer una educación general mínima de 8 a 10 años y proponerse como meta incorporar al sistema a todos los niños de edad escolar antes de 1999, de conformidad con las políticas educativas nacionales,

Todas estas acciones sirvieron de base para que en 1980, mediante el Decreto No 646 de fecha 13-6-1980 se creara el subsistema de Educación Básica: educación mínima de nueve años que se debe proporcionar a todo ciudadano, con la finalidad de estimular el desarrollo armónico de la personalidad y, que según el artículo $5^{\circ}$ de este decreto se enseñaría "a partir del año escolar 80-81 en áreas geográficas que previa investigación, hayan sido seleccionadas...”, y que progresivamente se extendería "con base en los resultados de la evaluación hasta generalizarse a nivel nacional”.

Ese Decreto (según Memoria y Cuenta que presentó el Ministro de Educación al Congreso Nacional de la República de Venezuela en sus sesiones de 1981), lo dictó el Ministerio de Educación para dar el esquema normativo y basamento legal a la Fase Experimental que inició su primera etapa en Octubre de 1980 en los grados $1^{\circ}$, 4o y 70 con una muestra de 18.952 alumnos y 250 maestros de 71 planteles, ubicados en seis entidades federales: Estado Aragua (15 planteles), Estado Falcón (8 planteles), Estado Zulia (14 planteles), Estado Monagas (9 planteles), Estado Táchira (12 planteles) y Estado Lara (13 planteles).

La incorporación progresiva de los planteles a nivel nacional continuó en el año escolar 1981-1982 con la incorporación de una muestra de 61.048 alumnos y 17.420 maestros de $1^{\circ}, 4^{\circ}$ y $7^{\circ}$ grado de 250 planteles correspondientes a las siete zonas que se inician en el Proyecto. Ellas con el Distrito Federal y los Estados Bolívar, Carabobo, Cojedes, Yaracuy, Sucre y Mérida. En esta segunda etapa del Proyecto en las seis zonas que se iniciaron en el año escolar 80-81
(Aragua, Falcón, Lara, Monagas, Táchira y Zulia), continuó la prosecución en los grados $1^{\circ}, 2^{\circ}, 4^{\circ}, 5^{\circ}, 70$ y $8^{\circ}$ grado.

En síntesis, el Nivel de Educación Básica se implantó oficialmente en forma de cascada, mediante la realización de dos fases, una de ensayo desde 1976 hasta 1984 y otra de instrumentación propiamente dicha de las tres etapas: la primera, de expansión y globalización en el año escolar 1985-1986; la segunda, de consolidación, en el período 1986-1987 y la tercera, de independización, entre 1987 y 1988.

Al extenderse y generalizarse ese nivel a todo el país, hubo la necesidad de eliminar algunas medidas, enfoques y acciones que se habían ensayado durante la ejecución del Proyecto Experimental de Educación Básica, entre ellas cabe destacar las siguientes:

- La reestructuración de los tres ciclos de 4,3 y 2 años respectivamente en tres etapas de tres años de duración cada una ( la primera etapa, comprendía $1^{\circ} 2^{\circ}$ y $3^{\circ}$ grado, la segunda: $4^{\circ}, 5^{\circ} 6^{\circ}$ grado y la tercera etapa: $7^{\circ}, 8^{\circ}$ y $9^{\circ}$ grado). Esta reestructuración resolvería los problemas que se habían suscitado en el ensayo, los cuales hacían muy difícil la utilización de las plantas físicas de las antiguas escuelas primarias para alojar los dos primeros ciclos, pues la incorporación del séptimo grado, requería de aulas no siempre disponibles (en algunos casos hubo la necesidad de cerrar secciones de primer grado para atender la prosecución de los alumnos que terminaban el sexto grado) y de equipos y de otros materiales que se hallaban instalados en los ciclos básicos de Educación Media, pero que no existían en los planteles donde se venía impartiendo la educación primaria. Todo esto traía como consecuencia la necesidad de construir edificaciones apropiadas que servirían de sede a las nuevas escuelas de nueve grado y dejar sin uso a los antiguos locales de las escuelas primarias y por ende constituiría un despilfarro enorme de recursos.

En sí, la división de la Escuela Básica que se adoptó y generalizó para todo el país entre 1985 y 1988 comprendió tres etapas, de las 
cuales las dos primeras siguieron funcionando en los planteles que ocupaban las escuelas primarias, mientras que la tercera etapa utilizó las edificaciones que ocupaban los antiguos ciclos básicos comunes.

En el establecimiento definitivo del nivel de Educación Básica, surgieron algunos inconvenientes relacionados con la atención pedagógica y académica de los docentes, debido en gran parte por vivencias experimentadas y por testimonios orales, el entrenamiento que recibió el personal docente conformado en su mayoría por maestros normalistas, algunos profesores y licenciados universitarios y maestros no graduados, fue muy breve y además no se propició la reflexión en profundidad sobre los criterios epistemológicos, sociológicos, psicológicos y pedagógicos del diseño, las bases doctrinarias y legales y sus implicaciones ideológicas, lo cual facilitó la aceptación sumisa y la reproducción acrítica del nuevo modelo.

Tampoco se crearon espacios que le permitiera a los docentes analizar su perfil profesional, desaprovechándose así la oportunidad para que sinceraran su fortalezas y debilidades y vincular a la vez sus experiencias y saberes previos con las exigencias de la Educación Básica.

\section{CAMBIOS CURRICULARES SIGNIFICATIVOS EN LA EDUCACIÓN BÁSICA 1979-1989}

Uno de los cambios significativos a nivel curricular en la Escuela Primaria Venezolana, lo constituye la creación del susbsistema de la Educación Básica, (Según Decreto No 646 emitido el 13 de Junio de 1980) y que a la vez fue consagrado por la Ley Orgánica de Educación de 1980 como segundo nivel del Sistema Educativo Venezolano. Al Ministerio de Educación introduce una serie de cambios e innovaciones en el Plan de Estudios con el propósito de generar un mejoramiento cualitativo de la educación en este nivel: los nueve años se organizaron en tres ciclos que se diferencian entre sí por la interrelación entre las unidades curriculares, los énfasis curriculares y los componentes afectivos.

Al Primer Ciclo, que comprendía los primeros cuatro años se le denominaba instrumental y de expansión y se caracterizaba por un alto nivel de interrelación entre las unidades curriculares, por el énfasis en lograr que el alumno manejara los instrumentos básicos para el proceso de aprendizaje como son la lectura, la escritura, la expresión oral y la habilidad de utilizar las operaciones elementales de cálculo para la resolución de problemas cotidianos sencillos, en consecuencia el énfasis era en la recreación, el área de Lengua y Matemática. Los conocimientos se presentaban en forma global e integrada, de allí que los contenidos eran presentados por áreas muy interrelacionadas unas de otras. Cada curso estaba a cargo de un solo maestro que dictaba todas las áreas, con excepción de aquellas muy especializadas como deporte, artes escénicas, entre otras.

El Segundo Ciclo: de consolidación, abarcaba el quinto, sexto y séptimo año de Educación Básica. El grado de interrelación entre las unidades curriculares era mediano, cada grupo de alumnos tenía como mínimo seis maestros y rotaba por igual número de aulas-ambientes. El énfasis estaba en los valores vinculados a la nacionalidad y al estímulo de sensibilidad social; de allí se desprende la importancia que se le daba al área de Pensamiento, Acción Social e Identidad Nacional.

El Tercer Ciclo : de independización, comprendía el octavo y noveno año y tenía como objetivo fundamental lograr una orientación general del alumno para su mejor adaptación a las circunstancias que le tocará vivir al salir del Nivel de Educación Básica. Se observaba un bajo nivel de interrelación entre las Unidades Curriculares y de las áreas entre sí. Se hacía un especial énfasis en la formación vocacional, dándole importancia a la formación para el trabajo, las ciencias y el autoaprendizaje. Evolución histórico-legal de la supervisión educativa 1979-1989. 
Según Decreto No 478 del 8 de enero de 1980, para fines de planificación y coordinación de las actividades de la Administración Pública Nacional, al igual que en el Decreto 1331 del 16 de diciembre de 1975, se establecen nueve regiones educativas, se mantienen sin modificar las regiones Centroocidental, Zuliana, Según Decreto No 478 del 8 de enero de 1980, para fines de planificación y coordinación de las actividades de la Administración Pública Nacional, al igual que en el Decreto 1331 del 16 de diciembre de 1975, se establecen nueve regiones educativas, se mantienen sin modificar las regiones Centroocidental, Zuliana, nororiental, Guayana e Insular. Se Crean las regiones Capital, Central y Llanos, modificándose las región de los Andes. Establecía además que en las regiones funcionaría un Consejo Regioneal de Desarrollo.

Con la Promulgación del Reglamento General de la Ley Orgánica de Educación, el 7/3/1986, quedan eliminadas las direcciones regionales de educación (DRE), según lo establecido en su artículo 156, que consagra definitivamente el Nivel Zonal, dando paso así a la consolidación definitiva de las Zonas Educativas en las veintitrés entidades federales del país, entre ellas la del Estado Lara como estructura administrativa dependiente del Ministerio de Educación, la cual según Resolución No 746 del 6/7/1988, se divide en 8 distritos escolares. 
Cuadro 1: Implantación de la Educación Básica,

Auge y Crisis del Proyecto

\begin{tabular}{|c|c|c|c|c|}
\hline Contexto Sociopolítico & $\begin{array}{l}\text { Caracterización del } \\
\text { Sistema Educativo }\end{array}$ & Aportes significativos & $\begin{array}{c}\text { Cambios Curriculares } \\
\text { significativos en la Educación } \\
\text { Básica }\end{array}$ & $\begin{array}{c}\text { Caracterización del } \\
\text { Sistema de } \\
\text { Supervisión } \\
\text { Educativa }\end{array}$ \\
\hline $\begin{array}{l}\text { 3.El clientelismo partidista invadió todos los sectores de la } \\
\text { actividad económica, las instituciones financieras, los ministerios } \\
\text { del poder ejecutivo y del poder judicial, fomentando la } \\
\text { incapacidad administrativa y la corrupción en el manejo de los } \\
\text { fondos. } \\
\text { 4.La crisis socioeconómica, profundizó las diferencias entre l os } \\
\text { grupos que conforman la sociedad venezolana, acelerando según } \\
\text { Rodríguez N (1998), por una parte la concentración de la riqueza } \\
\text { en grupos financieros y rentistas; y por la otra, generando el } \\
\text { empobrecimiento de la clase media e incrementando el porcentaje } \\
\text { de pobreza crítica a n } 43 \% \text {, lo cual trajo como consecuencia el } \\
\text { aumento } \\
\text { de los índices de deserción escolar, delincuencia juvenil, } \\
\text { mendicidad, drogadicción y otras forma de degradación del ser } \\
\text { humano, de donde se derivó una crisis ética, cuyo centro fue la } \\
\text { desvalorización de la dignidad humana, en contraartida a la } \\
\text { exaltación del poder y el dinero, sin importar los medios ara su } \\
\text { obtención. }\end{array}$ & $\begin{array}{l}\text {.Según la Ley Orgánica } \\
\text { de Educación de 1980, el } \\
\text { sistema educativo es un } \\
\text { conjunto orgánico } \\
\text { integrador de políticas y } \\
\text { servicios que garanticen } \\
\text { la unidad del proceso } \\
\text { educativo, tanto escolar } \\
\text { como extraescolar y su } \\
\text { continuidad a lo largo de } \\
\text { la vida de la persona } \\
\text { mediante un proceso de } \\
\text { educación permanente. } \\
\text { Comprende niveles y } \\
\text { modalidades. Son } \\
\text { niveles: educación } \\
\text { preescolar, que } \\
\text { constituye el primer } \\
\text { nivel; la educación } \\
\text { básica, el segundo nivel; } \\
\text { la educación media } \\
\text { diversificada y } \\
\text { profesional, el tercer } \\
\text { nivel; y la educación } \\
\text { superior, el cuarto nivel. } \\
\text { Son modalidades: la } \\
\text { educación especial, la } \\
\text { educación para las artes, } \\
\text { la educación militar, la } \\
\text { educación para la } \\
\text { formación de ministros } \\
\text { de culto, la educación de } \\
\text { adultos y la educación } \\
\text { extraescolar. } \\
\text { La Educación Básica } \\
\text { constituye el segundo } \\
\text { nivel obligatorio, tendrá } \\
\text { una duración de nueve } \\
\text { años y comprende tres } \\
\text { etapas de tres grados } \\
\text { cada una. La educación } \\
\text { media diversificada y }\end{array}$ & $\begin{array}{l}\text { 1..Se creó el Sistema de Servicios } \\
\text { Bibliotecarios Escolares según } \\
\text { Decreto No } 188 \text { del 27/06/79. } \\
\text { 2.Se creó el Seguro Escolar por } \\
\text { Decreto No } 357 \text { del 15/1/1979. } \\
\text { 3. Creación de la Educación } \\
\text { Básica de nueve grados con la } \\
\text { emisión del Decreto } 646 \text { el 13 de } \\
\text { Junio de 1980. } \\
\\
\text { 4..Promulgación de la Ley } \\
\text { Orgánica de Educación de 1980. } \\
\text { 5.El Ministerio de Educación } \\
\text { emite la resolución No 12 que } \\
\text { describe el perfil del docente en } \\
\text { atención a cinco roles básicos: } \\
\text { facilitador, investigador, } \\
\text { promotor social y administrador. } \\
\text { 6.El Ministerio de educación } \\
\text { conjuntamente con el Ministerio } \\
\text { de Estado para el desarrollo de la } \\
\text { Inteligencia, desarrollaron los } \\
\text { proyectos “Aprender a pensar”, } \\
\text { "Enriquecimiento instrumental” } \\
\text { “Ajedrez e Inteligencia” que } \\
\text { buscaban mejorar las destrezas } \\
\text { intelectuales de los estudiantes, } \\
\text { estimular la creatividad, } \\
\text { enriquecer la percepción y la } \\
\text { memoria, y despertar el deseo de } \\
\text { aprender y superación personal. }\end{array}$ & $\begin{array}{l}\text { 1. El Plan de Estudios de 1980, } \\
\text { comprendía tres ciclos. Al } \\
\text { primero se le denominaba } \\
\text { instrumental y de expansión y } \\
\text { comprendía los cuatro } \\
\text { primeros años, el énfasis } \\
\text { estaba en la lectura, la } \\
\text { escritura, la expresión oral y } \\
\text { las habilidades en las } \\
\text { operaciones matemáticas } \\
\text { básicas. El segundo ciclo, de } \\
\text { consolidación, abarcaba los } \\
\text { grados quinto, sexto y séptimo } \\
\text { año con énfasis en las áreas de } \\
\text { Pensamiento, Acción Social e } \\
\text { Identidad Nacional. El Tercer } \\
\text { Ciclo, de Independización, } \\
\text { comprendía el octavo y noveno } \\
\text { año, con énfasis en la } \\
\text { formación vocacional. } \\
\\
\text { 2.El Plan de estudios según } \\
\text { Resolución No } 590 \text { del } 3 \text { de } \\
\text { Julio de } 1987 \text {, correspondiente } \\
\text { al Nivel de Educación Básica } \\
\text { en sus tres etapas (la primera } \\
\text { que comprende los grados } 1^{\circ}{ }^{\circ} 2^{\circ} \\
\text { y 3 grado; la segunda, los } \\
\text { grados } 4^{\circ}, 5^{\circ} \text { y 6 } 6^{\circ} \text { grado y la } \\
\text { tercera, los grados } 7^{\circ}, 8^{\circ} \text { y } 9^{\circ} \\
\text { grado), estaba integrado por } \\
\text { siete áreas: 1).Lengua, que } \\
\text { comprendía las asignatura s } \\
\text { Castellano y Literatura e } \\
\text { Inglés. 2).,Matemática, que } \\
\text { comprendía la asignatura de } \\
\text { Matemática. 3) Ciencias de la } \\
\text { Naturaleza, y Educación para } \\
\text { la Salud, que comprende las } \\
\text { asignaturas: Estudio de la } \\
\text { Naturaleza, Educacín para la }\end{array}$ & $\begin{array}{l}\text { 1.Según Decreto no } \\
478 \text { del } 8 \text { de enero de } \\
1980 \text {, para fines de } \\
\text { planificación y } \\
\text { coordinación de las } \\
\text { actividades de la } \\
\text { Administración } \\
\text { Pública Nacional, al } \\
\text { igual que en el } \\
\text { Decreto } 1331 \text { del 16 } \\
\text { de diciembre de 1975, } \\
\text { se establecen nueve } \\
\text { regiones educativas, se } \\
\text { mantienen sin } \\
\text { modificar las regiones } \\
\text { Centroocidental, } \\
\text { Zuliana, nororiental, } \\
\text { Guayana e Insular. Se } \\
\text { Crean las regiones } \\
\text { Capital, Central y } \\
\text { Llanos, modificándose } \\
\text { las región de los } \\
\text { Andes. Establecía } \\
\text { además que en las } \\
\text { regiones funcionaría } \\
\text { un Consejo Regioneal } \\
\text { de Desarrollo. } \\
\\
\text { 2. Con la } \\
\text { Promulgación del } \\
\text { Reglamento General } \\
\text { de la Ley Orgánica de } \\
\text { Educación, el } \\
\text { 7/3/1986, quedan } \\
\text { eliminadas las } \\
\text { direcciones regionales } \\
\text { de educación (DRE), } \\
\text { según lo establecido } \\
\text { en su artículo 156, que } \\
\text { consagra } \\
\text { definitivamente el }\end{array}$ \\
\hline
\end{tabular}


6.La política social no facilitaba la transformación de la sociedad venezolana en un escenario de país moderno y productivo, sino por el contrario sólo se lograba paliar los efectos de la

ineficiencia y la improductividad de las empresas nacionales. Es decir tal como estaba concebida la política social, no era posible crear condiciones favorables hacia el trabajo y la productividad, dado que la relación Estado-Beneficiario, se reducía a un vínculo clientelista, paternalista y de dependencia. En sí, esa política, sólo estaba destinada a transferir incondicionalmente recursos a la población, mientras ocurría la modernización desde la vía conómica.

7. La concentración de los recursos y la ineslaticidad del gasto público en las áreas tradicionales de atención (educación y salud fundamentalmente) estaban muy vinculadas al gremialismo y al clientelismo existente en las instituciones del sector público, dentro de las agencias gubernamentales aprecen derechos adquiridos que dificultaban cualquier intento de cambio en la asignación de los recursos.

8.El crecimiento económico y la industrialización sobre las cuales se basaban las esperanzas de transformación y modernización del país durante el segundo quinquenio de esta tercera década dela democracia, no aportaron los resultados sociales deseados, generando una serie de medidas de austeridad pública

acompañada entre otras por el aumento del precio de la carne y de la gasolina, reorganización de cirtas empresas y la liquid ción de

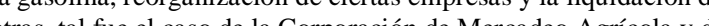
otras, tal fue el caso de la Corporación de Mercadeo Agricola y de la Corporación Venezolana de Fonento. Elinninación del subsidio a la producción láctea que trajo como consecuencia el aumento precio de estos productos (por ejemplo la leche pasteurizada ncrementó en un $120 \%$ su precio, pasando de un costo de 2,50 a 5,50 bolívares por litro)

8.En el segundo quinquenio de esta tercera década de la democracia se suscitaron una serie de hechos que conmocionaron la opinión pública nacional, tales como los pozos de la muerte, los jóvenes asesinados en Yumare y los desaciertos económicos que contribuyeron a la devaluación del bolívar a una paridad de más de 14 bolívares por dólar. profesional tendrá una duración no menor de dos años.La educación superior comprenderá la formación profesional y de post-grado.

Seún la Resolución No 590 del 3/7/1987, la educación básica comprende tres etapas:la primera que comprende los grados primero, segundo y tercero; la segunda los grados cuarto, quinto y sexto la tercera los grados séptimo, octavo y

noveno.
7.Se estimuló y promovió la práctica del deporte en los distintos niveles del sistema educativo a través de program escolares y programaciones especiales en coordinación con et IND.

8.Se designó según Resolución No 154 del 14/03/1985, la

Comisión Presidencial para el Estudio del Proyecto Educativo Venezolano (COPEN).Se reactivó el consejo Nacional de Educación.

10. Se estableció el Plan de Estudios correspondiente al Nivel de Educación Básica en sus tres etapas
Salud, Ciencias Biológicas, Física y Química 4) Estudio Sociales, que comprende als asignaturas de Historia de Venezuela, Cátedra

Bolivariana, Geografía de Educativas en las Venezuela, Educación Familiar federales del país, y Ciudadana, Historia Universal y Geografía General

6)Educación Estética, que comprende las asignaturas: Educación Musical y

Educación A tis

7) Educación para el Deporte y 7) Educación para el Trabajo .

entre ellas la del

Estado Lara como estructura administrativa dependiente de Ministerio de Educación, la cual según Resolución No 746 del 6/7/1988, se divide en 8 distritos escolares. 


\section{REFERENCIAS}

\section{Documentales}

CORDIPLAN (1971) IV Plan de la Nación 1970-1975. Caracas: Oficina Central de Coordinación y Planificación.

Ministerio de Educación Decreto 1331. (Nueva ordenación territorial: nueve regiones educativas). (Diciembre 16, 1975). Caracas: Autor.

Ministerio de Educación (1970) Decreto 478. ( División del territorio del país en Nueve regiones educativas) (Enero 8, 1970). Caracas: Autor

Ministerio de Educación. Ley orgánica de Educación de 1980. Gaceta Oficial de la República de Venezuela 2635 (extraordinaria). Julio 28, 1980. Caracas: Autor

Ministerio de Educación (1980) Educación Básica, Normativo. Caracas: Oficina Sectorial de Planificación y Presupuesto.

Decreto 646. Ministerio de Educación (Creación del subsistema de Educación Básica) (Junio 13, 1980) Caracas: Autor.

Memoria y Cuenta que presenta el Ministro de Educación al Congreso Nacional en las sesiones ordinarias de 1981. Caracas: Ministerio de Educación.

Ministerio de Educación (1982) Educación Básica. Plan de estudio. Diseño Experimental. 1982-1983 Caracas: Oficina Sectorial de Planificación y Presupuesto.

Ministerio de Educación Resolución No 12. (Gaceta Oficial de la República de Venezuela No 3085, de fecha 24/1/83)
Ministerio de Educación (1985) Educación Básica, una visión general. Caracas: Dirección de Educación.

Ministerio de Educación Resolución No 154. (Designación de la Comisión Presidencial para el Estudio del Proyecto Educativo Venezolano) Marzo 14, 1985. Caracas: Autor.

Ministerio de Educación VII Plan de la Nación (1984-1988) Proyecto Estratégico No 10. Caracas, Marzo, 1986. Caracas: Autor

Decreto 975. Ministerio de Educación. (Promulgación del Reglamento General de la Ley Orgánica de Educación. Gaceta Oficial No 3.713 Extraordinaria (Marzo 7, 1986)

Ministerio de Educación (1987) Educación Básica. Modelo Normativo. Plan de Estudio y Evaluación del Rendimiento Escolar. Caracas: Autor.

Resolución 746. Ministerio de Educación. (División de la Zona Educativa del Estado Lara en ocho distritos escolares). (Julio 6, 1988). Caracas: Autor.

UNESCO, CEPAL y OEA (1979) Declaración de México. Conferencia Regional de Ministros de Educación y de Ministros Encargados de la Planificación Económica de los Estados Miembros de la América Latina y del Caribe, México, D.F., 13 de Diciembre de 1979.

\section{Bibliográficas}


Albornoz, O. (1991) La Educación bajo el signo de la crisis. Volumen VI. Colección Educación. Caracas: Ediciones de la Biblioteca, UCV..

Alviárez, R (1987) La Democracia Venezolana. Criatura deforme. Caracas: Imprenta Universitaria de la UCV.

Fernández H (1981)Memoria de Cien Años. Historia de la Educación en Venezuela (9vols) Tomos V, VI (volumen I y II) Caracas: Ediciones del Ministerio de Educación.

(1982) Regionalización de la educación en Venezuela. Colección Ideas y Doctrinas sobre la Formación Profesional. Caracas: Instituto Nacional

de Cooperativa Educativa.

González Lucini F ( 1996) Temas Transversales y Áreas Curriculares. Madrid, España: Alauda- Anaya

Rodríguez, N (1991) La Educación Básica en Venezuela; Proyectos, realidad y perspectivas. Caracas: Ediciones Dolvia, C.A.

(1998) (Compiladora) Historia de la Educación Venezolana Caracas:Universidad Central de Venezuela, Facultad de Humanidades y Educación, Comisión de estudios de Postgrado.

Urbaneja D (1985) El sistema político o cómo funciona la máquina de procesar Decisiones. El caso venezolano. Caracas: Ediciones IESA.

\section{Citas y Referencias}

Garfinkel, L (1996) Ponencia presentada en el II Simposio de Descentralización de la Educación. Octubre 1996. Caracas: UPEL.

\section{Hemerográficas}

Ministerio de Educación y Organización de los Estados Americanos (OEA) (1985) Currículo. Revista Especializada para América Latina y el Caribe. No 16. Caracas. 